\title{
Depletion of the density of states at the Fermi level in metallic colossal magnetoresistive manganites
}

\author{
J. Mitra and A. K. Raychaudhuri \\ Department of Physics, Indian Institute of Science, Bangalore 560 012, India \\ Ya. M. Mukovskii and D. Shulyatev \\ Moscow State Steel and Alloys Institute, 075 Leninsky prospekt 4, Moscow 119049, Russian Federation
}

\begin{abstract}
We have performed tunneling spectroscopy measurements down to $4.2 \mathrm{~K}$ on single crystals of $\mathrm{La}_{0.75} \mathrm{Sr}_{0.25} \mathrm{MnO}_{3}$ and $\mathrm{La}_{0.7} \mathrm{Ca}_{0.3} \mathrm{MnO}_{3}$, which show the colossal magnetoresistive behavior in the ferromagnetic metallic phase. The tunneling spectroscopy measurements give information on the density of states (DOS) at and close to the Fermi level $E_{F}$. The observed energy dependence of the DOS is similar to that seen in correlated oxides with disorder, which is an important observation since it raises fundamental questions regarding the nature of the metallic state in these oxides. Measurements at low temperatures reveal that the DOS in these compounds show a depletion close to $E_{F},\left|E-E_{F}\right| \leqslant 15 \mathrm{meV}$; unlike that in conventional metals. The normalized DOS, close to $E_{F}$ shows an energy dependence $\propto\left|E-E_{F}\right|^{2}$ at low temperatures but remains finite as $E \rightarrow E_{F}$. The depth of the depletion is more in $\mathrm{La}_{0.7} \mathrm{Ca}_{0.3} \mathrm{MnO}_{3}$ compared to $\mathrm{La}_{0.75} \mathrm{Sr}_{0.25} \mathrm{MnO}_{3}$.
\end{abstract}

\section{INTRODUCTION}

The physics of colossal magnetoresistance (CMR) in hole doped rare-earth transition metal manganites with a generic formula $\mathrm{La}_{1-x} \mathrm{~A}_{x} \mathrm{MnO}_{3}$ is a topic of current interest. ${ }^{1}$ These oxides of the $\mathrm{ABO}_{3}$ perovskite structure originate from the parent compound $\mathrm{LaMnO}_{3}$. A mixed valency is created at the Mn site $\left(\mathrm{Mn}^{3+}, \mathrm{Mn}^{4+}\right)$ by substitution of La with divalent elements such as $\mathrm{Ca}$ or Sr. Though the parent compound is an antiferromagnetic insulator, above a critical doping $(x$ $\geqslant 0.2$ ) it gives rise to a ferromagnetic metallic (FMM) phase below the ferromagnetic transition temperature $T_{c}$ and a paramagnetic insulating (PMI) or paramagnetic metallic (PMM) phase above $T_{c}$. Our interests are in the distinct features in this low temperature FMM phase, which differ from those seen in conventional metals. For example, the FMM phase even in good single crystals have a relatively high residual resistivity. The conductivity $G$ is given by $G$ $\simeq e^{2} N_{s}\left(E_{F}\right) D$ [where $D$ is the electron diffusivity and $N_{s}\left(E_{F}\right)$ is the density of states (DOS) of the system at the Fermi level]. Thus, a low conductivity implies a low diffusivity for the carriers and/or a depleted DOS at $E_{F}$. From optical conductivity experiments it was observed that the Drude weight is considerably lower than what is estimated directly from the stoichiometric hole doping., ${ }^{2,3}$ On the other hand at low temperatures one finds the linear specific-heat coefficient $\gamma$, which is $\propto N_{s}\left(E_{F}\right)$ and is not much different from $\gamma_{\text {free-electron. }}{ }^{4,5}$ Thus a crucial question that one asks is what is the nature of the electronic DOS close to the Fermi level $E_{F}$, especially for the compositions deep into the FMM state. Since these states are those involved in the transport properties, a knowledge of these states is an essential ingredient in our understanding of the physics of these manganites. There is a need to look at the DOS near the $E_{F}$ carefully and to compare it with that in conventional metals. One of the important techniques to study $N_{s}(E)$ near the Fermi level is tunneling spectroscopy. Scanning tunneling spectroscopy measurements ${ }^{6,7}$ done on similar CMR systems have shown that $N_{s}\left(E_{F}\right)$ builds rapidly as $T$ is lowered below $T_{c}$, which indicates that the large increase in the conductivity of the system on cooling below $T_{c}$ can be attributed to the rapid increase of $N_{s}\left(E_{F}\right)$.

In this paper we report the tunneling spectroscopy (TS) measurements, carried out on single crystals of CMR manganites, $\mathrm{La}_{0.75} \mathrm{Sr}_{0.25} \mathrm{MnO}_{3}$ (LSMO) and $\mathrm{La}_{0.7} \mathrm{Ca}_{0.3} \mathrm{MnO}_{3}$ (LCMO) using barrier-type junctions. The measurements were carried down to $4.2 \mathrm{~K}$ with high-energy resolution, which allowed us to study $N_{s}(E)$ very close to $E_{F}(\mid E$ $\left.-E_{F} \mid<0.1 \mathrm{eV}\right)$.

\section{EXPERIMENTAL DETAILS}

The LSMO and LCMO samples were grown by the floatzone technique, details of which are published elsewhere. ${ }^{8}$ Figure 1 shows the resistivity data for the samples. LSMO, which is a wide bandwidth system, undergoes a PMM-FMM phase transition with $T_{c} \sim 342 \mathrm{~K}$. It remains metallic till 4.2 $\mathrm{K}$ with a residual resistivity, $\rho_{o}(\mathrm{LSMO})=41.6 \mu \Omega \mathrm{cm}$. LCMO, which is a medium bandwidth system, has a PMIFMM phase transition with $T_{c} \sim 250 \mathrm{~K}$ and also remains metallic with $\rho_{o}(\mathrm{LCMO})=161 \mu \Omega \mathrm{cm}$. The resistivities were measured by the standard four-probe method with the lowfrequency ac technique. The TS experiments were done in a standard bath-type cryostat. The cryostat is fitted with a precision screw that carries the tip, which is the counter electrode of the junctions. This screw arrangement allows us to control the pressure on the tip-sample junction and hence the junction resistance $R_{J}$, enabling us to study junctions of various resistances with this setup. A thermometer fitted next to the sample reads the temperature. The single crystals' surfaces were mechanically scraped and polished just before loading them into the cryostat. The cryostat was subsequently evacuated and purged with $\mathrm{He}$ gas before cooling 


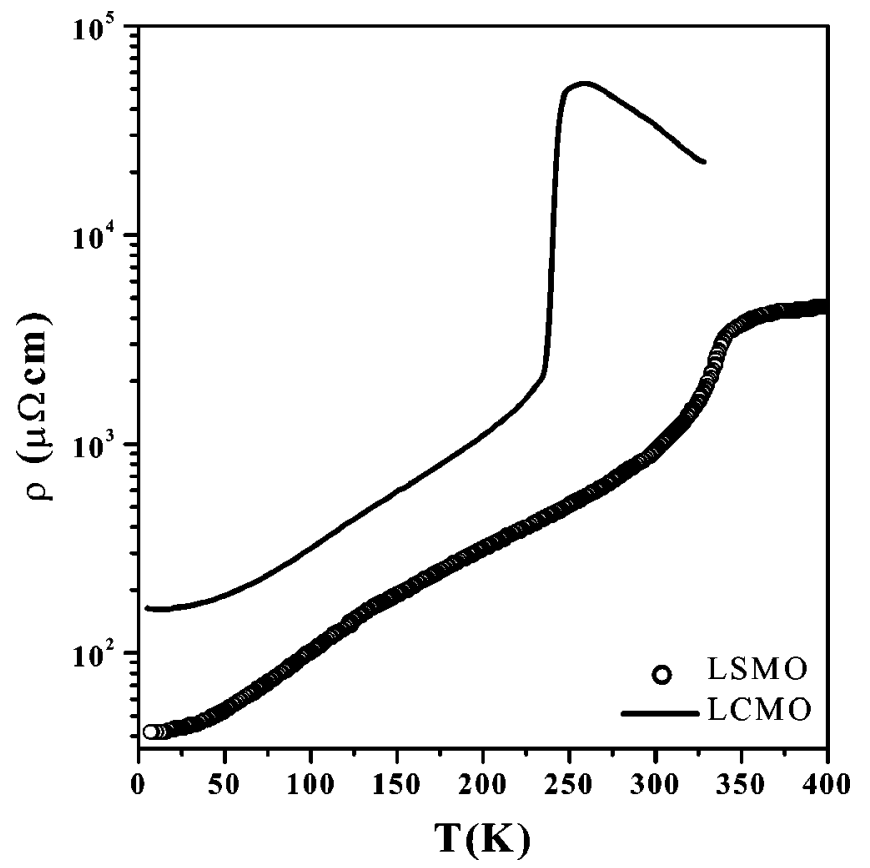

FIG. 1. Resistivity vs temperature of $\mathrm{La}_{0.75} \mathrm{Sr}_{0.25} \mathrm{MnO}_{3}$ and $\mathrm{La}_{0.7} \mathrm{Ca}_{0.3} \mathrm{MnO}_{3}$.

down. The experiments were carried out either in the evacuated chamber at different fixed temperatures or by dipping the junctions directly into liquid He. One set of tunnel junctions was formed between the sample and $\mathrm{Pb}$ as the counter electrode, the native oxide on $\mathrm{Pb}$ serving as the tunnel barrier. A superconducting material was chosen as the counter electrode because the observation of the superconducting energy gap is an evidence of the formation of the tunnel junction. As a check that the observed results do not depend on the nature of the junctions, another set of junctions were studied between etched Au tips and sample in the spear anvil geometry. In case of the Au tunnel junctions data was taken for different junction resistances. All the tunneling curves $(d I / d V-V)$ of the junctions were measured by the ac modulation technique. When measurements are made with the bias within tens of $\mathrm{mV}$ and the absolute changes in conductances $d I / d V$ are only a few percent, the ac modulation technique gives a higher signal-to-noise ratio compared to the standard dc technique, where conductance $(d I / d V-V)$ curves are obtained by numerical differentiation of the $I-V$ curves. Experimentally, we apply a constant amplitude ac modulation voltage $\delta V \cos (\omega t)$ riding over a dc bias, $V_{d c}$, across the junction, $V=V_{d c}+\delta V \cos (\omega t)$ with $\delta V \ll V_{d c}$ (typical values of $\delta V \simeq 100-200 \mu V)$. The dc bias across the junction determines the energy of the electron crossing the barrier. The dc bias is given by an extremely low-frequency triangular wave from a synthesizer and is added to the synthesized sine wave signal from a DSP based lock-in amplifier by a mixer cum current booster circuit. The lock-in amplifier also measures the ac component of the current across the junction that gives the conductance of the junction. A Taylor series expansion of the mixed current across the junction gives

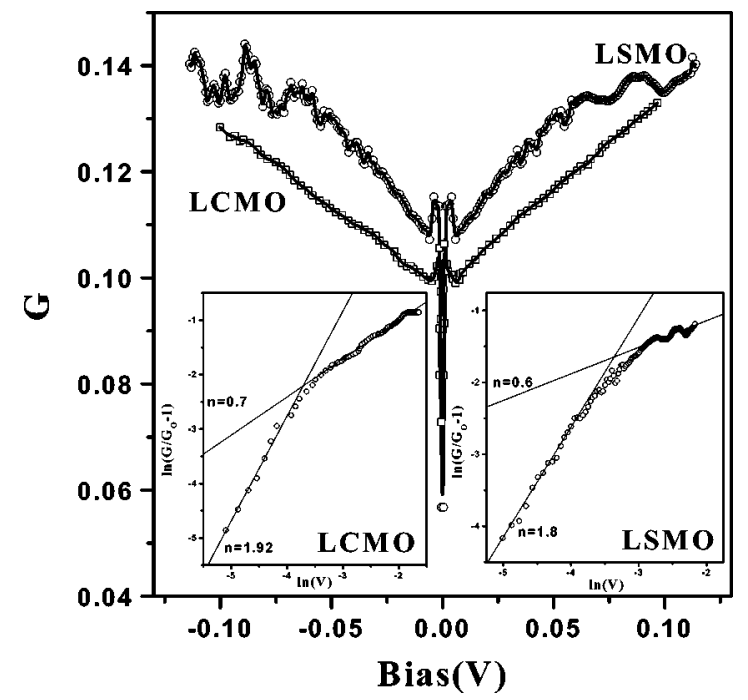

FIG. 2. Tunneling conductance for Pb-LSMO and Pb-LCMO junctions. The insets showing the respective $\ln \left(G / G_{O}-1\right)-\ln V$ plots, their fits to Eq. (2) and the sharp change over of the exponent $n$.

$$
\begin{aligned}
I(t)= & I_{d c}+(d I / d V) \delta V \cos (\omega t) \\
& +\frac{1}{2}\left(d^{2} I / d V^{2}\right) \delta V^{2} \cos ^{2}(\omega t)+\cdots,
\end{aligned}
$$

where $I_{d c}$ is the dc component of the current. The ac voltage measured (at frequency $\omega$ ) across a standard resistor $\mathbf{R}_{v}$ (that lies in series with the junction) gives the amplitude of the second term in Eq. (1), which gives the dynamic conductance $d I / d V$ of the junction. The $d I / d V$ and the dc voltage across the junction were measured simultaneously using the lock-in amplifier and a dc voltmeter, respectively. Thus as $V_{d c}$ is varied across the junction we record $d I / d V$ as a function of bias. For each $R_{J}$ and temperature the experiment was repeated over 5 cycles and finally averaged for further calculations.

To minimize heating of the junctions the TS data at $4.2 \mathrm{~K}$ was taken with the junctions directly dipped in liquid He. The higher-temperature data was taken with the temperature stabilized at certain given temperatures with $\mathrm{He}$ exchange gas around the junction. The tunnel junctions studied, especially those with Au electrodes, are of relatively low resistances. Heating of the junctions prevented us from going to larger bias. For a $20-\Omega$ junction the dissipation at the junction is $20 \mu \mathrm{W}$ with a current of a few milliamphere and that for a $500-\Omega$ junction is $0.8 \mu \mathrm{W}$. The higher- $R_{J}$ junctions were too noisy though we could attain stable junctions of few $k \Omega s$.

\section{RESULTS AND DISCUSSION}

\section{A. Tunneling conductance}

Figure 2 shows typical conductance curves for Pb-LSMO and $\mathrm{Pb}$-LCMO junctions taken at $4.2 \mathrm{~K}$. The superconduct- 
ing gap in the tunneling data can be seen for both samples. A comparison of these conductance curves with scanning tunneling spectroscopy studies between platinum electrodes ${ }^{7}$ show that they do not follow the normal barrier tunneling behavior that one observes in the case of conventional metalinsulator-metal tunneling. The data show a dip in the tunneling conductance at low bias and a flattening at higher bias. Interestingly, such a behavior has been found even in a number of other correlated metallic oxides. ${ }^{9}$ In some of these oxides the tunnel conductance follows a more generic dependence on bias as in Eq. (2) with $n \simeq 0.5-0.8$ and is $<1$. Note that this dip does not refer to the sharp dip occurring close to zero bias in Fig. 2, which occurs due to the superconducting gap of $\mathrm{Pb}$. To quantify the above statement we fitted the tunneling conductance to the relation,

$$
G(V)=G_{o}\left[1+\left(\frac{|V|}{V^{*}}\right)^{n}\right]
$$

where $V^{*}$ and $n$ are parameters that are determined from the fit of the conductance data to the above equation. The insets of Fig. 2 show the $\ln \left(G / G_{o}-1\right)$ vs $\ln (V)$ plot for LSMO and LCMO. The region $|V| \leqslant \Delta_{P b} \simeq 2.5 \mathrm{meV}$ is not included in this plot and in subsequent calculations because the conductance in this region is dominated by features in the DOS of the superconducting $\mathrm{Pb}$. From the plots and their fits to Eq. (2) we find that a single exponent $n$ cannot be used over the whole bias range but it shows a sharp crossover near $V$ $\simeq 15 \mathrm{meV}$. For LSMO the exponent $n \approx 1.8$ for $V$ $<15 \mathrm{meV}$ and $n \approx 0.6$ for $V \geqslant 15 \mathrm{meV}$, while for LCMO $n$ $\approx 1.9$ for $V<15 \mathrm{meV}$ and $n \approx 0.7$ at higher bias. As a check that the results do not depend on the nature of the counter electrode and to probe the low-bias regime, $V \leqslant 20 \mathrm{mV}$ more thoroughly, another set of junctions were made with $\mathrm{Au}$ as the counter electrode. Figure 3 shows the scaled dynamic conductance $\left(G / G_{o}\right)$ vs $V$ for LSMO and LCMO, taken with a Au tip. $G_{o}$ is the conductance at zero bias. For LSMO, the data were taken varying $R_{J}$ from $32 \Omega$ to $300 \Omega$, while for LCMO it was varied from $18 \Omega$ to $400 \Omega$. The observed tunneling curve (scaled by $G_{o}$ ) becomes nearly $R_{J}$ independent for $R_{J} \geqslant 200 \Omega$. In subsequent analysis we have taken tunneling curves for junctions with $R_{J} \geqslant 200 \Omega$. The $\ln \left(G / G_{o}-1\right)$ vs $\ln (V)$ plots (insets in Fig. 3) for both samples show that the exponent $n$ turns out to be $\simeq 2$ near zero bias and $\simeq 0.7$ for $|V| \geqslant 13 \mathrm{meV}$ in good agreement with those seen in the Pb junctions (see Fig. 3). Thus the voltage dependence of the tunneling conductance is not dependent on the counter electrode. The exponent of $n \simeq 0.8$ in these CMR manganites in the FMM state has been reported previously. ${ }^{10}$ However the sharp changeover of the exponent to $n \simeq 2$ near zero bias has not been observed before.

In this context we recall that it has been known that in disordered conductors the effect of electron-electron interaction can be quite prominent. These systems with strong electronic correlations have significantly modified DOS at $E_{F}$. Theoretical calculations show that on the metallic side the DOS near $E_{F}$ is modified as (Ref. 11),
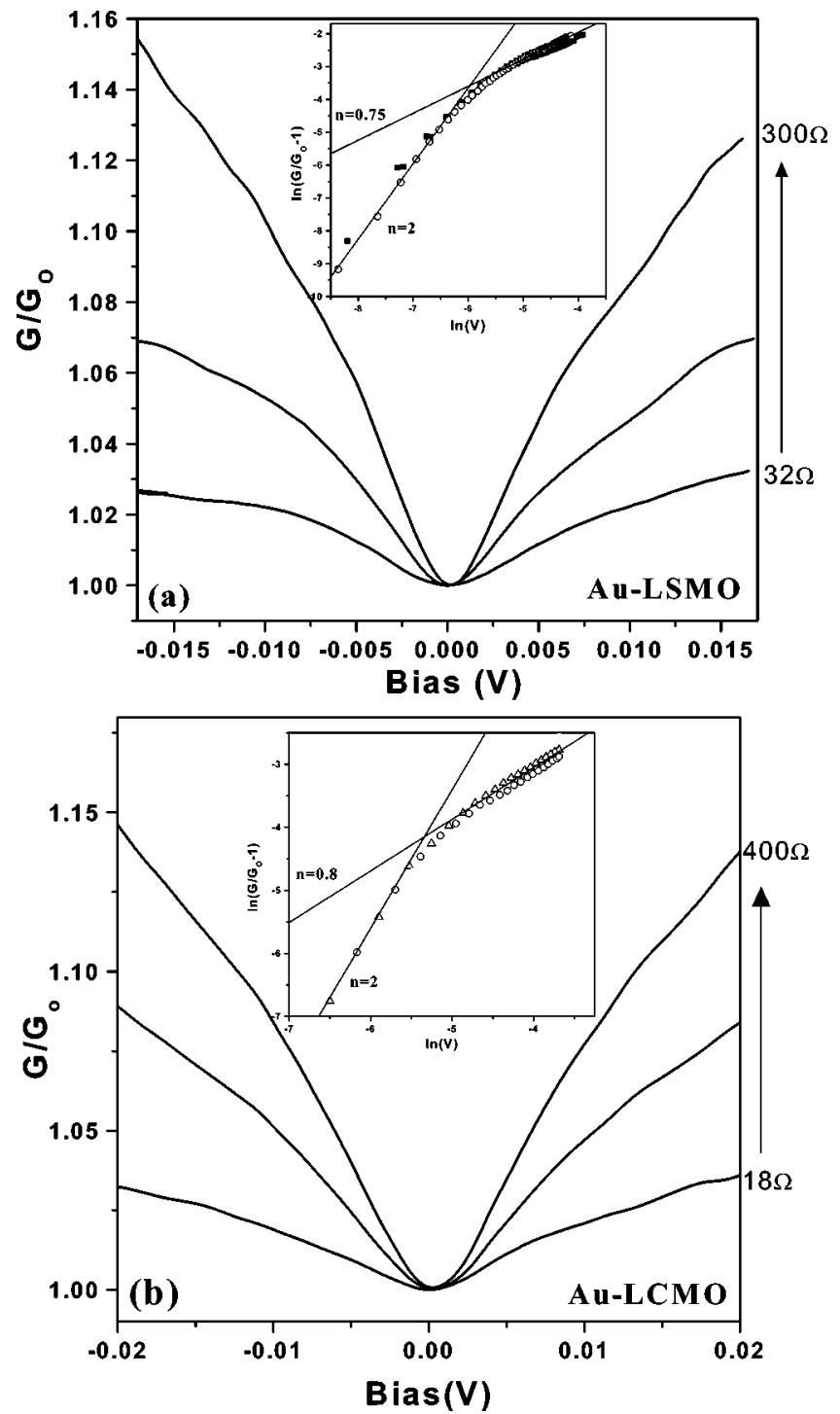

FIG. 3. Scaled conductance for (a) Au-LSMO and (b) AuLCMO junctions. The respective insets showing the $\ln \left(G / G_{O}-1\right)$ $-\ln V$ plot to emphasize the sharp change in the exponent from $n$ $\simeq 2$ to $n \simeq 0.7$.

$$
N_{s}\left(E-E_{F}\right)=N_{s}\left(E_{F}\right)\left[1+\left(\frac{\left|E-E_{F}\right|}{\Delta}\right)^{\xi}\right],
$$

where $\Delta$ is a constant, $N_{s}\left(E_{F}\right)$ is the DOS at the Fermi level, and $\xi=0.5$. The second term in the above equation arises due to the modifications to the DOS due to many-body effects. The theory also predicts that $\Delta$ decreases as the electron diffusivity $D$ decreases following relation $\Delta \propto D^{3} \propto \sigma^{3}$, where $\sigma$ is the conductivity of the system. Generally, the value of the exponent $\xi=0.5$ in weakly localized systems, while in more disordered systems (in correlated oxide metals) the exponent is larger $(0.5-0.8)$ and $\xi \rightarrow 1$ at the metal insulator transition. ${ }^{9}$

The observed tunneling conductance $G(V)$ is approximately proportional to $N_{s}\left(E-E_{F}\right)$, as explained later. The dependence of $G(V)$ on $V$ as expressed through Eq. (2) can 


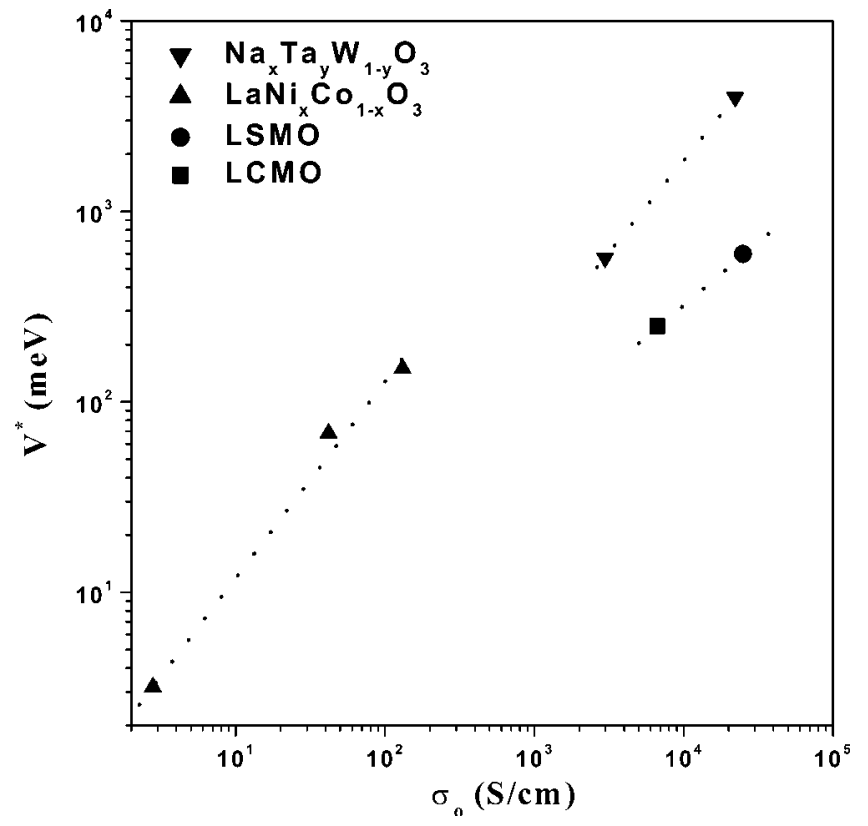

FIG. 4. Dependence of corelation gap $V^{*}$ on residual conductivity of $\mathrm{LaNi}_{x} \mathrm{Co}_{1-x} \mathrm{O}_{3}, \mathrm{Na}_{x} \mathrm{Ta}_{y} \mathrm{~W}_{1-y} \mathrm{O}_{3}$, LSMO and LCMO.

be interpreted as the validation of $N_{s}\left(E-E_{F}\right)$ following Eq. (3) with the identification of exponent $n=\xi$ and the energy scale $V^{*}=\Delta$. In these samples $n \simeq 0.7-0.8$ for $|V|$ $\geqslant 15 \mathrm{meV}$, which is indicative of the presence of significant electron-electron interactions. The further changeover of $n$ to 2 near $V=0$, as we show below is indicative of the presence of a pseudogap in the DOS near $E_{F}$. The fits to the conductance data with Eq. (2) give us an estimate of the parameter $V^{*}$, which can be interpreted as the scale of the interaction and a measure of $\Delta / e$ [Eq. (3)]. The value of $V^{*}$ is $\sim 250 \mathrm{mV}$ for LCMO, while that for LSMO $V^{*}$ is $\sim 600 \mathrm{mV}$. It is also rather interesting to note that the values obtained here are remarkably similar to those obtained from tunneling studies on other metallic perovskite oxides ${ }^{9}$ such as $\mathrm{LaNi}_{x} \mathrm{Co}_{1-x} \mathrm{O}_{3}$ and $\mathrm{Na}_{x} \mathrm{Ta}_{y} \mathrm{~W}_{1-y} \mathrm{O}_{3}$. Figure 4 shows $V^{*}$ as a function of $\sigma_{o}$ for LSMO and LCMO along with those for the oxides mentioned above, for different doping and thus different $\sigma_{o}$. Here $\sigma_{o}$ is the residual conductivity, a measure of the disorder in the system. In all these oxides we find that $V^{*} \propto \sigma_{o}$. Though the other transition metal oxides are not manganites and may have very little in common with these CMR compounds, the low-temperature tunneling studies yield similar values for the parameter $V^{*}$ and similar dependence on $\sigma_{o}$. All these oxides belong to the $\mathrm{ABO}_{3}$ class of perovskite oxides with a narrow conduction band formed by the hybridization of the $3 d$ orbitals of the transition metal ions with $2 p$ orbitals of the intervening oxygen. Again in most of these oxides, the charge carriers though itinerant, lie very close to the metal-insulator transition. Presumably this leads to similar correlations in these systems.

The above analysis tells us how the tunneling conductance varies with $V$ and reflects upon the DOS, but they do not give us the exact variation of the DOS with energy. In the following section we address this question of deconvoluting the conductance data to extract the DOS.

\section{B. Estimation of energy dependence of $N_{s}(E)$ from tunneling conductance}

In order to get the exact DOS and its energy dependence, we have used the following model and extracted the $N_{s}(E)$ as outlined below. The dependence of tunneling conductance on bias mainly arises from two sources: (i) the intrinsic energy dependences of the DOS of the sample $\left[N_{s}(E)\right]$ and the counter electrode/tip $\left[N_{t}(E)\right]$ and (ii) the energy dependence of the quantum-mechanical barrier penetration factor $\bar{t}$. While deconvoluting the conductance data to extract $N_{s}(E)$ we assume that $N_{t}(E)$ is flat within the energy range of our experiment $\left[\left(E-E_{F}\right) \leqslant 0.5 \mathrm{eV}\right]$. Experimental and theoretical calculations show that both $\mathrm{Au}$ and $\mathrm{Pb}$ have flat $\mathrm{DOS}$ for $\left|E-E_{F}\right| \leqslant 0.5 \mathrm{~V} .{ }^{12,13}$ More nontrivial, however, is to remove the contribution of the barrier penetration factor $\bar{t}$ while deconvoluting the data. The standard technique followed in TS is to compute $d \ln (I) / d \ln (V)$ assuming that this takes care of the said contribution from the barrier factor. The assumption is that the parameter $I / V$ gives us a reasonable estimate of $\bar{t}$ as found empirically ${ }^{14,15}$ in many practical cases thereby removing the barrier contribution when we compute $(d I / d V) /(I / V)$. This scaled dynamic conductance $d \ln (I) / d \ln (V)$ has its own limitations. It is only valid when the energy dependence of the barrier function is stronger than that of the DOS. ${ }^{16}$ Further it does not eliminate the effect of the barrier parameter completely and the quantity becomes undefined whenever a gap opens up in the DOS. We have used an alternate deconvoluting procedure to normalize the data and obtain the DOS, which is briefly described below. ${ }^{16}$ The tip-sample tunneling current (in the symmetric form), across a barrier, at a finite temperature is given by,

$$
\begin{aligned}
I(s, V, W, T)= & c \int_{-\infty}^{\infty} N_{s}\left(E+\frac{e V}{2}\right) N_{t}\left(E-\frac{e V}{2}\right) \bar{t}(s, E, W) \\
& \times\left[f\left(E-\frac{e V}{2}\right)-f\left(E+\frac{e V}{2}\right)\right] d E,
\end{aligned}
$$

where $E$ is the energy of the electron, $c$ is a constant dependent on the tip-sample effective junction area, $s$ is the tipsample distance, $W$ is the average work function of the tip and sample surface, $V$ is the bias between the tip and the sample, $T$ is the temperature, $N_{t}(E)$ and $N_{s}(E)$ are the tip and sample DOS, and $f(E)$ is the Fermi function at temperature $T$. The barrier penetration factor $\bar{t}$ is estimated by a WKB semiclassical approach. We model the barrier as an one-dimensional trapezoidal barrier and its tunneling probability calculated in the WKB approximation gives $\bar{t}$ as

$$
\bar{t}(s, E, W)=\exp (-2 k s \sqrt{2(W-E)})
$$

where $k=\sqrt{m} / \hbar$. In the above expression all the energies are measured with respect to $E_{F}$ which is the zero of the energy scale. Equation (4) can be simplified at low temperatures, $k_{B} T \ll \mathrm{eV}$ ( $k_{B}$ is Boltzmann's constant), by using a step function approximating the Fermi-Dirac distribution function. We 


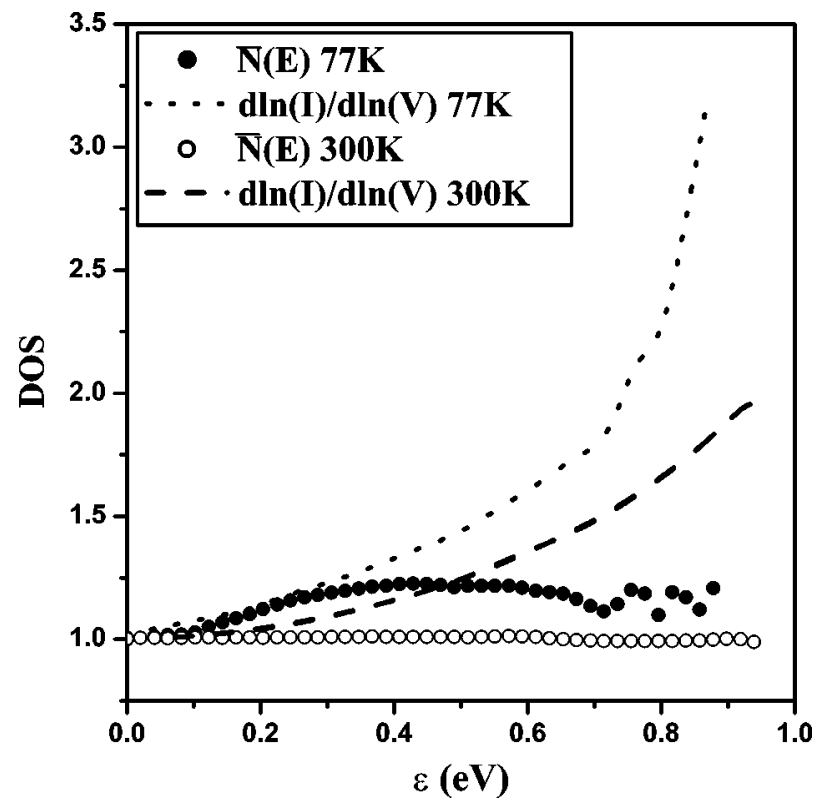

FIG. 5. The DOS calculated for LCMO by two different normalization techniques at $77 \mathrm{~K}$ and at $300 \mathrm{~K}$. The $I / V$ normalization data give a larger energy dependence due to the incomplete removal of the barrier parameter $\bar{t}$. The energy scale is $\epsilon=E-E_{F}$.

define the tunneling current $I_{\text {const }}^{0}$ for a constant tip and sample DOS $\left[N_{t}(E)=N_{t}\right.$ and $\left.N_{s}(E)=N_{s}\right]$ by the relation

$$
I_{\text {const }}^{0}(s, V, W)=c N_{t} N_{s} \int_{-e V / 2}^{e V / 2} \bar{t}(s, E, W) d E,
$$

$$
\begin{aligned}
\frac{d I_{\text {const }}^{0}}{d V}=\sigma_{\text {const }}^{0}(s, V)= & \frac{c}{2} N_{t} N_{s}(\bar{t}(s, e V / 2, W) \\
& +\bar{t}(s,-e V / 2, W)) .
\end{aligned}
$$

Equation (7) gives us the differential tunneling conductance for constant DOS. It clearly shows that the bias dependence of the functions $I_{\text {const }}^{0}$ and $\sigma_{\text {const }}^{0}$ arises solely from that of the barrier function $\bar{t}$. With the above approximations we fit the bare conductance data with Eq. (7) and estimate the barrier parameters $W$ and $s$. This fit essentially gives us an estimate of $\bar{t}$ and takes care of the broad features of the tunneling data, particularly, in the high-bias regime. Thus dividing the bare data by the estimated $\bar{t}$ removes the barrier effect and gives us a normalized conductance. Thus the observed energy dependence of the normalized conductance arises solely from the sample DOS and its energy dependence, which we henceforth refer to as the normalized DOS $[\bar{N}(E)]$. The subtleties of this technique and details are discussed elsewhere. ${ }^{16}$ Though this method of obtaining the DOS gives the correct energy dependence of $N_{s}(E)$ it does not give the correct absolute value of the same. By using Pt, which has a flat $N_{s}(E)$ in the energy range of interest, we have checked that the tunneling curve is mainly defined by the barrier factor. ${ }^{7}$ A constant DOS as in Pt gives a parabolic tunneling conductance $(G \propto V)$.

In Fig. 5 we have plotted the DOS for LCMO at 77 and $300 \mathrm{~K}$, calculated by the previous method of normalizing with $I / V$ [i.e., $d(\ln I) / d(\ln V)]$ and that according to the present method $[\bar{N}(E)]$. The results calculated from the two

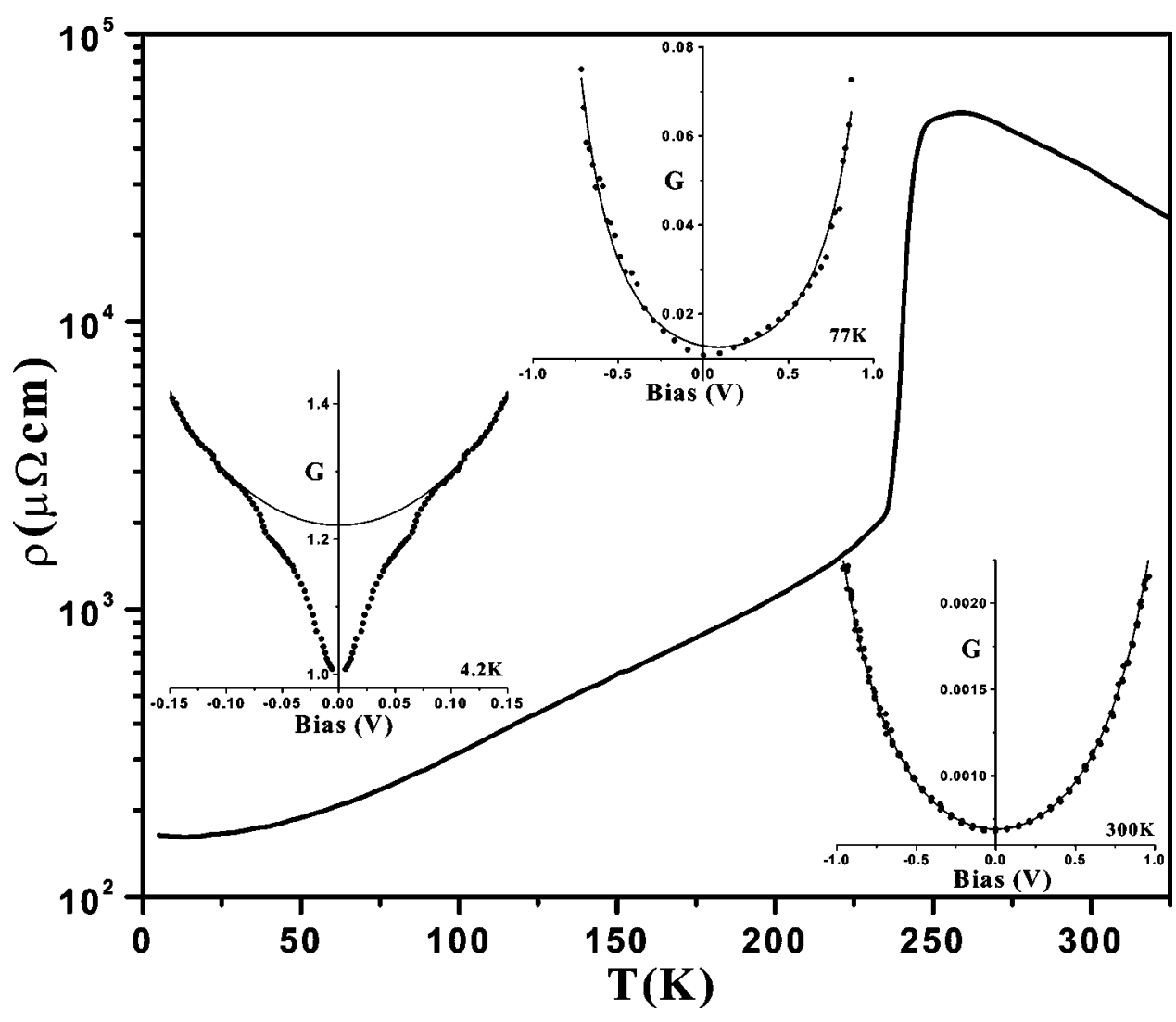

FIG. 6. The temperature variation of resistivity of LCMO and tunnel conductance $G(d I / d V)$ for $\mathrm{Pb}-\mathrm{LCMO}$ junction. The solid lines showing the fit for $\sigma_{\text {const }}^{o}$ at the respective temperatures as outlined in the text. 


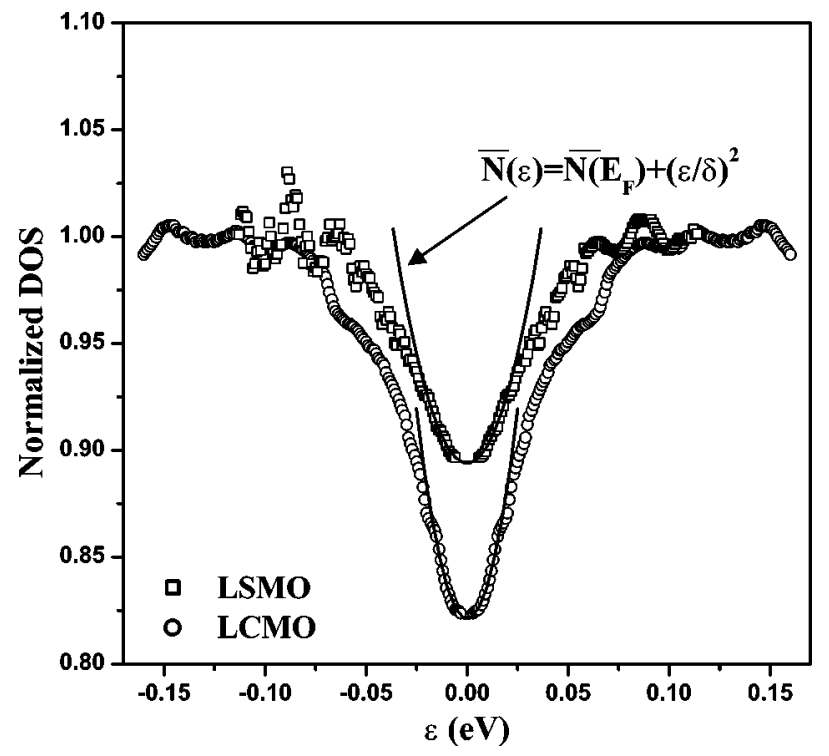

FIG. 7. The deconvoluted DOS for LCMO and LSMO at $4.2 \mathrm{~K}$ from $\mathrm{Pb}$-LSMO and $\mathrm{Pb}$-LCMO junctions. The solid lines close to $E_{F}$ show the fit to $N(\epsilon)=N\left(E_{F}\right)+(\epsilon / \delta)^{2}$ at low bias.

methods are different, particularly at higher bias, $V$ and hence at higher energy $\epsilon$ (where $\epsilon=E-E_{F}$ ). The scaled DOS calculated from the $I / V$ normalization retain much of the energy dependence of the barrier, which shows up as a steep rising DOS at higher energy. This factor is removed to a large extent in the method followed here. In the discussion that follows we will only refer to $\bar{N}(E)$.

\section{Temperature dependence of tunneling conductance and DOS}

In order to study temperature dependence of the tunneling conductance and the DOS, the tunneling experiment was carried out at representative temperatures for $4.2 \mathrm{~K}<T$ $<300 \mathrm{~K}$, with a Pb-LCMO junction. Figure 6 shows the evolution of the bare conductance curves with temperature for LCMO. The solid line in each case indicates the best fit to Eq. (7), which takes care of the barrier parameter. It has been observed that at temperatures below $T_{c}$ the tunneling conductance curves are weakly asymmetric unlike those at $300 \mathrm{~K}$. An inherent asymmetry in $d I / d V-V$ is expected due to the trapezoidal nature of the barrier. In our theoretical calculations outlined earlier the assumed work function $W$ $=\left(W_{1}+W_{2}\right) / 2$, thus we had assumed an average value of the tip and sample work functions. In order to explain the weak asymmetry of the conductance data we modified the fit equation (7) suitably to incorporate $W_{1}$ and $W_{2}$ as,

$$
\sigma_{\text {const }}^{0}(s, V)=\frac{c}{2} N_{t} N_{s}\left[\bar{t}\left(s, e V / 2, W_{1}\right)+\bar{t}\left(s,-e V / 2, W_{2}\right)\right] .
$$

As an example, for the conductance curve at $T=77 \mathrm{~K}$ we obtained the best fit values of $W_{1}=0.9 \pm 0.1 \mathrm{eV}$ and $W_{2}$ $=1.2 \pm 0.1 \mathrm{eV}$. The two work functions are different but close. With this modified expression we were able to fit the conductance curves at the intermediate temperatures and obtain the DOS as a function of temperature. Figure 7 shows the normalized DOS for LCMO and LSMO adopting the above normalization procedure at $4.2 \mathrm{~K}$. It is clear from the figure that there is a finite depletion in the DOS near $E_{F}$ (small $\epsilon$ ) for both the samples. The depletion in the DOS for the wider band gap LSMO is shallower than that for LCMO. We term this depletion as a pseudo gap (PG). For both the samples, LSMO and LCMO the deconvoluted DOS fit a function of the form $\bar{N}(\epsilon)=\bar{N}\left(E_{F}\right)+(\epsilon / \delta)^{2}$, near $\epsilon=0$. It is this quadratic dependence of $N_{s}(E)$ on $\epsilon$ that gives rise to $n \simeq 2$ for $|V| \leqslant 15 \mathrm{meV}$, when we fitted the measured $G(V)$ to Eq. (2). The fit parameter $\delta$ for $\mathrm{LSMO} \simeq 110 \mathrm{meV}$ and that for $\mathrm{LCMO} \simeq 80 \mathrm{meV}$. Such a depletion in DOS though very shallow is unconventional in a metallic system. We note that this depletion is not observed at higher temperature and shows up only below $20 \mathrm{~K}$. Since the strong energydependent features, like the depletion in the DOS, occur below $15 \mathrm{meV}$ it is understandable that these features start showing up only at low temperatures. At higher temperatures the thermal smearing $\left(\approx 3 k_{B} T\right)$ suppresses such small energy features.

\section{Comparison with other experiments}

We would like to put our present investigation in the context of past tunneling studies. There are reported TS studies done on manganites particularly, close to the metal-insulator transition (MIT) temperature, using scanning tunneling spectroscopy (STS). ${ }^{7,17,18}$ The first temperature-dependent TS studies close to the MIT using STS was done by a previous investigation from our group. ${ }^{7}$ It was observed that a gap in the DOS opens up at $E_{F}$ close to $T_{c}$ and closes as the sample is cooled below $\mathrm{T}_{c}$ leading to a finite DOS at $E_{F}$. A comparison of the present conductance data above $100 \mathrm{~K}$ with the above investigation ${ }^{7}$, which was carried down till $100 \mathrm{~K}$, shows that they are very similar though they have been taken by different types of tunnel junctions. Spatially resolved STS images close to $T_{c}$ were subsequently reported by Fath et al. ${ }^{17}$ on single crystals and films of $\mathrm{La}_{1-x} \mathrm{Ca}_{x} \mathrm{MnO}_{3}(x$ $=0.33$ ). They observed coexisting metallic and insulating phases close to $T_{c}$. At low temperatures $T \ll T_{c}$ the sample had predominantly metallic regions with a finite DOS near $E_{F}$. Interestingly, even in this metallic state there were small yet finite fractions of insulating phases. The tunneling curves observed by us in the region $T<T_{c}$ are similar to those observed in this investigation. In a recent study on LSMO films (grain size $\approx 45 \mathrm{~nm}$ ) the spatial inhomogeneity of conductance close to the transition temperature was investigated. ${ }^{18}$ The results observed are similar to that of Fath et al. ${ }^{17} \mathrm{Al}-$ though spatially resolved tunneling conductance was measured there was no explicit discussion on change of the tunneling curve as a function of $T$. Interestingly, they also observed filamentlike insulating phases in the metallic regime $(T=87 \mathrm{~K})$. In view of the brief discussion we find that the tunneling conductance (and hence DOS) has not been probed by previous studies at low enough temperatures to closely look into the low-bias region $(|V|<0.1 \mathrm{eV})$ in the 
metallic phase. In particular, the shallow dip opening up below $15 \mathrm{meV}$ could not be seen till $T<50 \mathrm{~K}$. In contrast to past studies the present investigation (done with bulk barrier) does not have the spatial resolution inherent in STM based STS investigations, although, it gives a much stabler junctions which can be effectively cooled to $4.2 \mathrm{~K}$ in order to explore the small bias regime. The tunneling DOS that we report is thus an averaged spectrum. However, in the temperature region of interest to us $T \ll T_{c}$, all the past STM based tunneling studies have shown that the metallic phase is by far the most prevalent phase. In addition, since there is a gap in the DOS of the insulating phase it will make negligible contribution to the total tunneling conductance. It is thus expected that what we observe primarily give the characteristics of the metallic phase.

The occurrence of a shallow depletion in the DOS in a conventional metallic system is rather unusual. The observation of such a depletion is thus an interesting issue. A pseudogap in the DOS at $E_{F}$ has been observed in bilayer manganites by angle resolved photoemission spectroscopy experiments carried down to $10 \mathrm{~K} .{ }^{19}$ In these systems (though they are metallic) there is vanishing spectral weight of the DOS at $E_{F}$. In cubic (three-dimensional) manganites like $\mathrm{La}_{1-x} \mathrm{Sr}_{x} \mathrm{MnO}_{3}$ (with $x \geqslant x_{c} \approx 0.2$ ) though there is a finite spectral weight at the Fermi level, below the MIT, it is unusually low. ${ }^{20,21}$ However, these photoemission experiments were done above $80 \mathrm{~K}$ and with their energy resolution $(>0.15 \mathrm{eV})$, we will not be able to probe the region where we see the small gap opening up. The optical conductivity experiments, ${ }^{2,3}$ as mentioned before finds a Drude-like conductivity although the Drude weight is much smaller than that expected from the carrier concentration. All these experiments point towards the anomalous nature of the DOS in these materials in the ferromagnetic metallic phase below $T_{c}$. Our observation that though the DOS is finite there is a noticeable depletion at low-energy range close to $E_{F}$ may explain some of the anomalies such as low Drude weight and high resistivity in these materials.

\section{E. Physical mechanism for the pseudogap formation}

In the following we suggest some of probable scenarios that may explain the anomalous nature of the DOS. The dependence of the observed DOS on Eq. (3) for $|\epsilon|>15 \mathrm{meV}$ with $\xi \geqslant 0.5$ already points to the existence of significant correlation effects as has been seen in a number of correlated metallic oxides close to the insulator metal transition. ${ }^{9}$ In addition we observe the PG. The quadratic dependence of $\bar{N}(\epsilon)$ on $\epsilon$ for $|\epsilon|<15 \mathrm{meV}$ is suggestive of a Coulomb gap as seen in many insulators. ${ }^{22}$ In disordered insulators a soft gap opens up and a long-range Coulomb interaction $\left(\alpha^{\prime} / r\right)$ leads to $\bar{N}\left(E_{F}\right) \rightarrow 0$. However, such effects occur in electronic systems that are substantially disordered and often in the insulating side of the critical region of metal-insulator transition. Thus a Coulombic origin of the pseudo gap may not be the most likely possibility.

Recently, it has been shown that in manganites the ground state can be a phase separated state with coexistence of microscopic clusters of antiferromagnetic (insulating) and ferromagnetic (conducting) phases. ${ }^{23}$ In the region of phase separation, model calculations/simulations show that at $T$ $=0$ around the chemical potential there is a PG with the DOS $\propto|\epsilon|^{m}$ with $m>1$. In this case the PG tags the chemical potential. In three-dimensional $\mathrm{La}_{1-x} \mathrm{Ca}_{x} \mathrm{MnO}_{3}$ and $\mathrm{La}_{1-x} \mathrm{Sr}_{x} \mathrm{MnO}_{3}$ these models predict that the phase separation and the PG formation occurs below a certain carrier concentration, which is the low doping regime $(x \leqslant 0.2)$. In the concentration range of our samples $x \approx 0.25-0.4$ such microscopic phase separation leading to a PG is not expected in the parameter space explored so far. ${ }^{23}$ However, given the small value of the gap one cannot rule out similar mechanisms to be operative.

Another alternative scenario is the existence of polaronic states. The metallic state in these materials can have localized dynamic Jahn-Teller distortions. Electrical conductivity shows clear evidence that strong electron-phonon interaction is present in these manganites. ${ }^{24,25}$ Such interactions can lead to localized polarons that can bind a fraction of the carriers in shallow localized states leading to a dip in the DOS and PG formation. This will depend on the width of conduction band and is expected to be more effective in systems with narrower band (and lower $T_{c}$ ). Indeed, we find that the depth of the gap is more in LCMO (in which the polaronic effects are more pronounced) than in LSMO.

To summarize, we have carried out detailed tunneling experiments on single crystals of LSMO and LCMO, which show CMR. We analyzed the tunneling data using a model of the barrier in order to find the energy dependence of the DOS, $N(E)$, from the tunneling data. We found that for $|\epsilon|$ $\leqslant 15 \mathrm{meV}$ there is a depletion of the DOS unlike conventional metals. This depletion being a low-energy phenomena shows up clearly only at low temperatures. As outlined above there are different interactions that can lead to such a depletion or a shallow pseudogap DOS at $E_{F}$.

\section{ACKNOWLEDGMENTS}

J.M. acknowledges CSIR (Government of India) for financial support. A.K.R. wants to thank D.S.T. (Government of India) for a sponsored scheme. The work was done under India-Russia ILTP program.
${ }^{1}$ CMR, Charge Ordering and Related Properties of Manganese Oxides, edited by C.N.R. Rao and B. Raveau (World Scientific, Singapore, 1998); Colossal Magneto-resistive Oxides, edited by Y. Tokura (Gordon and Breach Science, Netherlands, 2000).
${ }^{2}$ Y. Okimoto, T. Katsufuji, T. Ishikawa, A. Urushibara, T. Arima, and Y. Tokura, Phys. Rev. Lett. 75, 109 (1995).

${ }^{3}$ Y. Okimoto, T. Katsufuji, T. Ishikawa, T. Arima, and Y. Tokura, Phys. Rev. B 55, 4206 (1997). 
${ }^{4}$ J.J. Hamilton, E.L. Keatly, H.L. Ju, A.K. Raychaudhuri, V.N. Smolyaninova, and R.L. Greene, Phys. Rev. B 54, 14926 (1996).

${ }^{5}$ T. Okuda, A. Asamitsu, Y. Tomioka, T. Kimura, Y. Taguchi, and Y. Tokura, Phys. Rev. Lett. 81, 3203 (1998).

${ }^{6}$ Amlan Biswas and A.K. Raychaudhuri, J. Phys.: Condens. Matter 8, L739 (1996).

${ }^{7}$ Amlan Biswas, Suja Elizabeth, A.K. Raychaudhuri, and H.L. Bhat, Phys. Rev. B 59, 5368 (1998).

${ }^{8}$ D. Shulyatev et al., J. Cryst. Growth 198, 511 (1999).

${ }^{9}$ A.K. Raychaudhuri, K.P. Rajeev, H. Srikanth, and N. Gayathri, Phys. Rev. B 51, 7421 (1995).

${ }^{10}$ Ashutosh Tiwari and K.P. Rajeev, Phys. Rev. B 60, 10591 (1999).

${ }^{11}$ B.L. Altshuler and A.G. Aronov, in Electron-Electron Interactions in Disordered Systems, edited by A. L. Efros and M. Pollak (North-Holland, Amsterdam, 1985).

${ }^{12}$ E.L. Wolf, in Principles of Electron Tunneling Spectroscopy (Oxford University Press, New York, 1985).

${ }^{13}$ P.G. Collins, J.C. Grossman, M. Ct, M. Ishigami, C. Piskoti, S.G. Louie, M.L. Cohen, and A. Zettl, Phys. Rev. Lett. 82, 165 (1999).

${ }^{14}$ N.D. Lang, Phys. Rev. B 34, 5947 (1986).

${ }^{15}$ J.A. Stroscio and R.M. Feenstra, in Scanning Tunneling Micros- copy, edited by J.A. Stroscio and W.J. Kaiser, Methods of Experimental Physics Vol. 27 (Academic, New York, 1993).

${ }^{16}$ Vladimir A. Ukraintsev, Phys. Rev. B 53, 11176 (1996).

${ }^{17}$ M. Fath, S. Friesem, A.A. Menovsky, Y. Tomioka, J. Aarts, and J.A. Mydosh, Science 285, 1540 (1999).

${ }^{18}$ T. Becker, C. Streng, Y. Luo, V. Moshnyaga, B. Damaschke, N. Shannon, and K. Samwer, Phys. Rev. Lett. 89, 237203 (2002).

${ }^{19}$ D.S. Dessau, T. Saitoh, C.H. Park, Z.X. Shen, P. Villella, N. Hamada, Y. Moritomo, and Y. Tokura, Phys. Rev. Lett. 81, 192 (1998).

${ }^{20}$ D.D. Sarma et al., Phys. Rev. B 53, 6873 (1996).

${ }^{21}$ J.-H. Park, C.T. Chen, S-W. Cheong, W. Bao, G. Meigs, V. Chakarian, and Y.U. Idzerda, Phys. Rev. Lett. 76, 4215 (1996).

${ }^{22}$ A.L. Efros, V.L. Ngnen, and B.I. Shlovskii, J. Phys. C 12, 1869 (1979).

${ }^{23}$ A. Moreo et al., Phys. Rev. Lett. 83, 2773 (1999); 84, 5568 (2000).

${ }^{24}$ Guo-meng Zhao, V. Smolyaninova, W. Prellier, and H. Keller, Phys. Rev. Lett. 84, 6086 (2000).

${ }^{25}$ J. Mitra, A.K. Raychaudhuri, N. Gayathri, and Ya.M. Mukovskii, Phys. Rev. B 65, 140406 (2002). 